\title{
A DETERMINATION OF THE MASSES OF THE FIVE OUTER PLANETS
}

\author{
R. L. DUNCOMBE, W. J. KLEPCZYNSKI, and P. K. SEIDELMANN \\ U.S. Naval Observatory, Washington, D.C., U.S.A.
}

\author{
(Presented at IAU Colloquium No. 9, 'The IAU System of \\ Astronomical Constants', Heidelberg, Germany, August 12-14, 1970.)
}

\begin{abstract}
Planetary masses are determined from an extensive analysis of observations of the five outer planets and of seven selected minor planets.
\end{abstract}

The values of the masses of Jupiter, Saturn, Uranus, and Neptune currently adopted for use in the national ephemerides are essentially those determined by Newcomb (1898). The adopted mass of Pluto is based on that of Wylie (1942). These values of the planetary masses were incorporated in the simultaneous numerical integration of the orbits of the five outer planets performed by Eckert et al. (1951) which forms the basis for the ephemerides printed in the American Ephemeris (U.S.A.) and in the Astronomical Ephemeris (U.K.). Their integrations were adjusted to extended series of observations which terminated in 1938.

Despite the relative recency of the Eckert et al. investigation we were led to take up the present study of the orbits and the masses of the outer planets for the following reasons. First, observations of Neptune at the present time are diverging from the predicted position of the planet by nearly 4 arc seconds in longitude. Second, the gravitationally determined value of the mass of Pluto by Wylie of 0.91 Earth masses when combined with the direct measure of the diameter of Pluto of $5928 \mathrm{~km}$ yields an unacceptably large value of the mean density of Pluto of at least $40 \mathrm{~g}$ per cubic centimeter. Finally, the accumulation of $30 \mathrm{yr}$ of precise meridian circle observations of Jupiter, Saturn, Uranus, and Neptune beyond those utilized by Eckert, et al. appeared to make such a study worthwhile.

The investigation began with the study of the motion of Neptune, because of the apparent failure of past theories to represent observations very far removed in time from the observations to which the constants of the theory were adjusted. Newcomb's theory (1899), amended to include the effect of Pluto with reciprocal mass 360000 , was adjusted to observations in 1795 and from 1846-96. By 1938 this theory failed to represent observations in orbital longitude by over $5 \mathrm{arc} \mathrm{sec}$. The present numerical theory of the motion of Neptune by Eckert $e t$ al. incorporating the same reciprocal mass of Pluto, and fitted to observations in 1795 and from 1846-1938, fails to represent the observed longitude of Neptune at the present epoch. This led us to investigate the orbit of Neptune on the possibility that an adjustment to the mass of Pluto might be required.

The analysis involved the simultaneous numerical integration of the equations of motion for the five outer planets which were then fitted to observations over extended 
periods of time, including the modern epoch. With the exception of Jupiter, the mass of each planet was determined by its effect on the motion of the planet immediately interior to it. The mass of Jupiter quoted here stems from a separate investigation of its effect on the motion of seven minor planets of the Hecuba group. Since introduction of revised mass values into the analysis often required the repetition of some steps of the analysis, it is not feasible to present the results in the order in which they were derived. Rather, the results for the planetary masses are presented commencing with Jupiter and moving out through the solar system.

The present study of the mass of Jupiter rests on an analysis of seven minor planets whose longitudes experience a long period perturbation ( 70 to $90 \mathrm{yr}$ ) due to the action of Jupiter. The residual amplitude of this long period term, as shown by observations, is a fairly sensitive index of the correction to the adopted mass of Jupiter. Essentially, for each minor planet a numerical integration of the equations of motion was performed and then fitted to observations from discovery up to the present time. Based on these adjusted elements a second numerical integration was performed and again fitted to observations, but this time by an adjustment to the mass of Jupiter in addition to the correction of the orbital elements of the minor planet.

TABLE I

Mass of Jupiter

$\begin{array}{lll}\text { Minor planet } & \text { Reciprocal mass } & \text { Mean error } \\ & & \\ 10 & 1047.351 & 0.006 \\ 24 & 1047.359 & 0.010 \\ 31 & 1047.372 & 0.006 \\ 52 & 1047.351 & 0.027 \\ 48 & 1047.340 & 0.024 \\ 57 & 1047.350 & 0.004 \\ 65 & 1047.387 & 0.004\end{array}$

The first four values shown in Table I were derived by Klepczynski(1969) from minor planets 10, 24, 31 and 52. The others stem from investigations of minor planets 48,57 and 65 by Zielenbach (1968), Fiala (1968), and O'Handley (1967). The weighted mean of these 7 determinations gives a reciprocal mass of Jupiter of $1047.366 \pm 0.006$ mean error. Although the mean seems quite reasonable, the scatter amongst the individual determinations is disconcerting and undoubtedly indicates the presence of systematic errors.

For the investigation of the motions of Jupiter, Saturn, Uranus, and Neptune, the observational data given by Eckert et al. (1951) were used. Their data were augmented, and in some cases replaced, by U.S. Naval Observatory meridian circle observations extending from 1913 to 1968 for Jupiter and Saturn and from 1938 to 1968 for Uranus and Neptune. For Pluto, the observations from 1914 to 1965 discussed by Cohen et al. (1967) were utilized. 
For each of several trial values of the reciprocal mass of Saturn, shown in Table II, a numerical integration of the equations of motion for all five outer planets was performed by Cowell's method. An orbit adjustment was made to the observations of all five outer planets, a new integration performed and a final orbit adjustment made. The orbit rectification for Jupiter and Saturn rests on their respective observations for the period 1913-68 although comparison was made with the observations from 1781 forward. Two classes of solution were made separately. One, a six-unknown solution for corrections to the orbital elements of Jupiter; the other, a seven-unknown solution for corrections to the orbital elements of Jupiter, and simultaneously for a

Table II

Reciprocal mass of Saturn

\begin{tabular}{llll}
$\begin{array}{l}\text { Reciprocal } \\
\text { mass test } \\
\text { values }\end{array}$ & $\begin{array}{l}\text { Jupiter (1913-1968) } \\
\Sigma v^{2}(\operatorname{arc~sec)}\end{array}$ & $\begin{array}{l}\text { Reciprocal } \\
\text { mass (seven- } \\
\text { unknown } \\
\text { solution) }\end{array}$ \\
\hline 3497.7 & 109.54 & 125.75 & 3498.7 \\
3498.7 & 107.45 & 125.68 & 3498.7 \\
3499.7 & 109.60 & 125.59 & 3498.7 \\
\hline
\end{tabular}
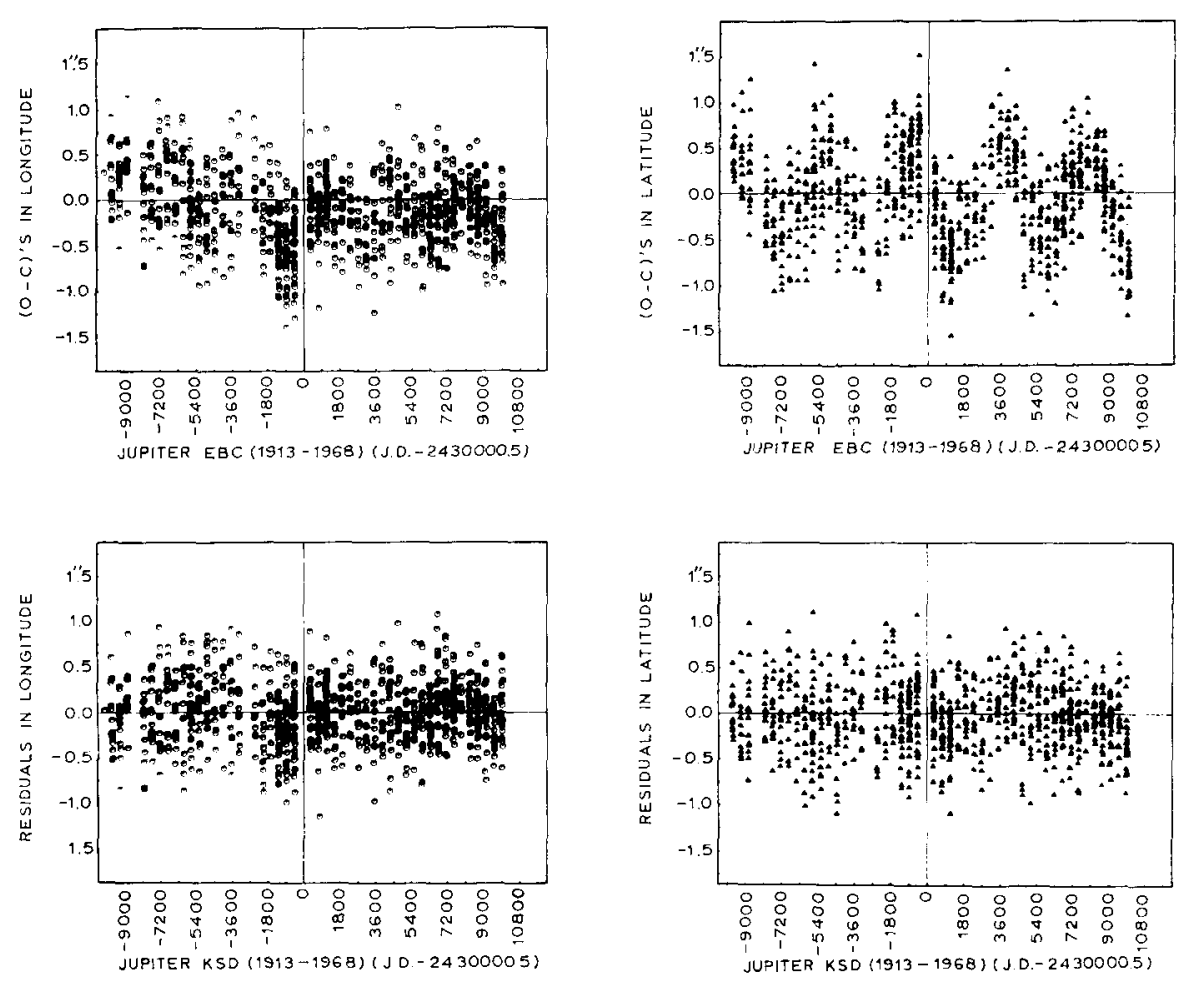

Fig. 1. 
correction to the mass of Saturn. The sums of the squares of the residuals in orbital longitude and latitude resulting from the six-unknown solutions, for the several trial values of the reciprocal mass, are shown in Table II. The values of the reciprocal mass determined from the seven-unknown solutions of the same observational material are in the final column. The value of the reciprocal mass of Saturn, determined from the seven-unknown solutions, is $3498.7 \pm 0.2$ mean error (Klepczynski et al., 1970). Differentiating the equation of the parabola fitted through the three values of the sums of the squares of the longitude residuals yields the same reciprocal mass. The improvement effected in representing the Jupiter observations is evident in Figure 1.

TABLE III

Reciprocal mass of Uranus

\begin{tabular}{llcl}
\hline $\begin{array}{l}\text { Reciprocal } \\
\text { mass test } \\
\text { values }\end{array}$ & \multicolumn{2}{l}{$\begin{array}{l}\text { Saturn (1913-68) } \\
\Sigma v^{2}(\operatorname{arc~sec)}\end{array}$} & $\begin{array}{l}\text { Reciprocal } \\
\text { mass (seven- } \\
\text { unknown } \\
\text { solution) }\end{array}$ \\
\cline { 2 - 3 } 22538 & $\lambda$ & $\beta$ & \\
22764 & 146.16 & 168.16 & 22700 \\
22795 & 144.99 & 168.07 & 22692 \\
22869 & 145.47 & 168.04 & 22691 \\
22693 & 146.81 & 167.95 & 22689 \\
\hline
\end{tabular}
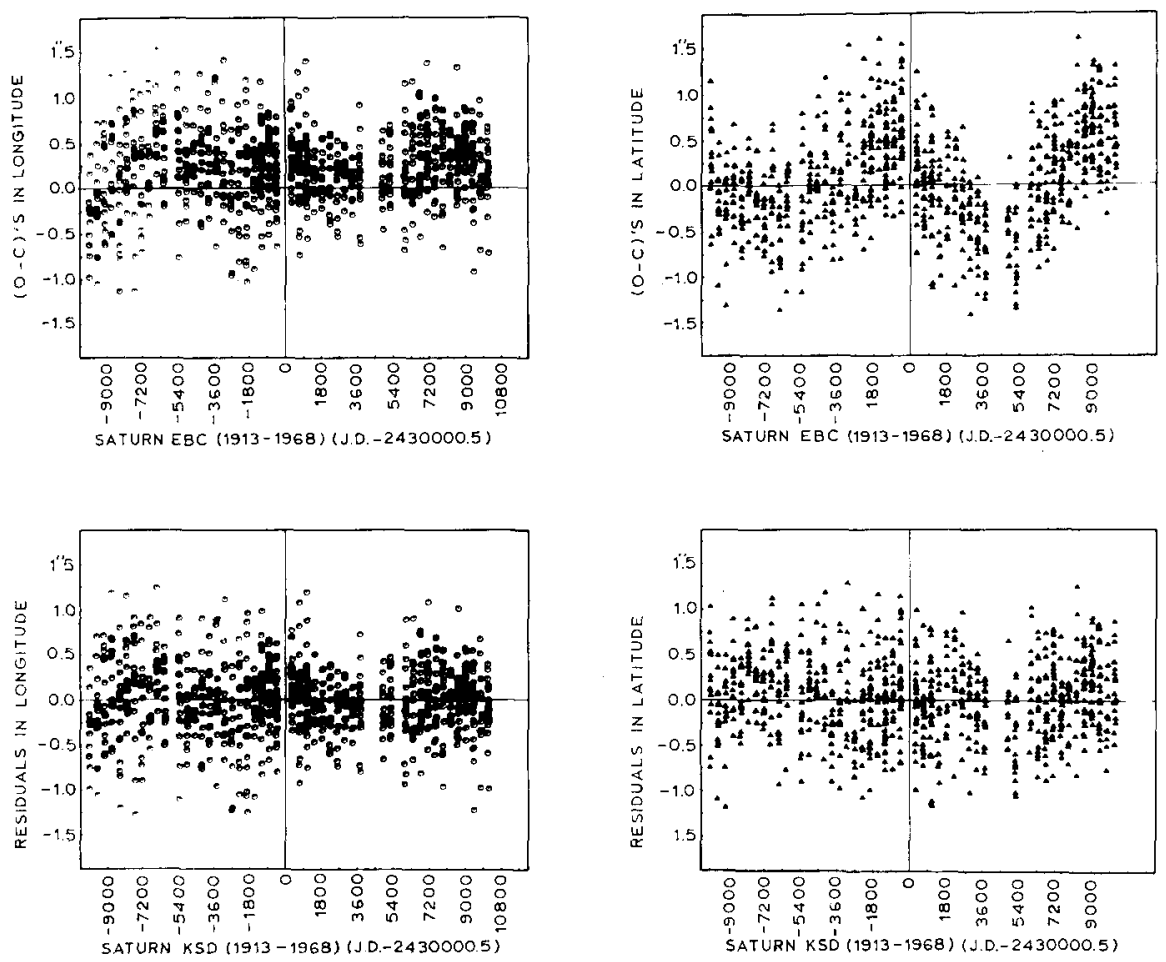

Fig. 2. 
Adopting the new mass of Saturn, several trial values for the mass of Uranus were selected and the same procedure was followed to determine the mass of Uranus from the motion of Saturn. Table III shows the several trial values of the reciprocal mass of Uranus, as well as the sums of the squares of the residuals in orbital longitude and latitude resulting from the six-unknown solutions utilizing those trial values. The reciprocal mass of Uranus given by the seven-unknown solution is $22692 \pm 33$ mean error (ibid.). As a check, differentiating the equation of the parabola fitted through the sums of the squares of the longitude and latitude residuals for the first four values given in the table indicates a reciprocal mass of 22691. The improved agreement with the observations utilizing the new orbit of Saturn is shown in Figure 2.

Using the new mass of Uranus, several trial values for the mass of Neptune were selected (Table IV) and the same procedure was followed to determine the mass of Neptune from the motion of Uranus. Table IV gives the sums of the squares of the residuals $\left(\Sigma v^{2}\right)$ in orbital longitude and latitude resulting from the six-unknown solutions of the observations from 1781 to 1968 . The values of the reciprocal mass determined from the seven-unknown solutions of the same observations are also shown in Table IV. Differentiating the equation of the parabola fitted through the sums of the squares of the longitude residuals indicates a reciprocal mass of 19346 . The average of this value with the mean of the two seven-unknown solutions is 19349 .

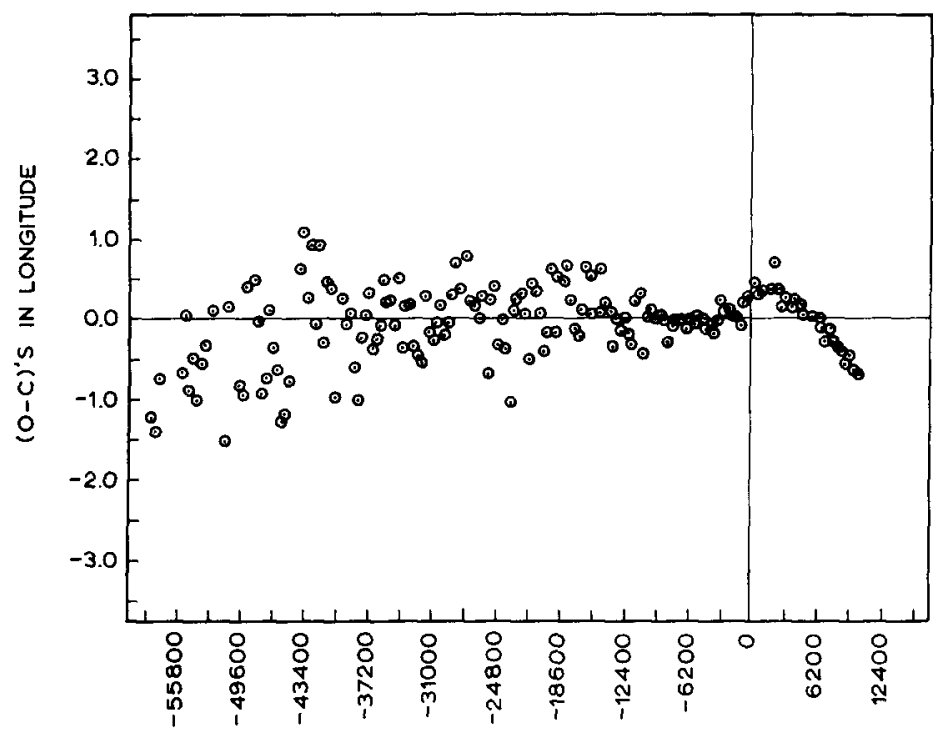

Fig. 3. Uranus, five outer planets (JD-2 430 000.5).

Adopting this as a test value in a new integration the seven-unknown solution yielded a value for the reciprocal mass of Neptune of $19349 \pm 28$ mean error (Seidelmann et al., 1969). The improvement in the representation of the longitude observations pachieved by the new orbit is illustrated by Figure 3 (the observations comared to the 
Eckert et al., theory) and Figure 4 (the observations compared to the present theory). Adopting this mass of Neptune and choosing several trial values for the mass of Pluto the procedure is repeated. This time, however, the solution is complicated by the relatively short arcs through which Neptune and Pluto have moved since the discovery of Neptune (approximately $270^{\circ}$ for Neptune and $75^{\circ}$ for Pluto). Because of this, the seven-unknown solution is unable to discriminate between corrections to the

TABLE IV

Reciprocal mass of Neptun

\begin{tabular}{lrrl}
$\begin{array}{l}\text { Reciprocal } \\
\text { mass test } \\
\text { values }\end{array}$ & \multicolumn{2}{l}{$\begin{array}{l}\text { Uranus (1781-1968) } \\
\sum v^{2}(\operatorname{arcsec})\end{array}$} & $\begin{array}{l}\text { Reciprocal } \\
\text { mass (seven- } \\
\text { unknown } \\
\text { solution) }\end{array}$ \\
& $\lambda$ & $\beta$ & \\
18800 & 887.55 & 85.12 & \\
19234 & 95.38 & 87.24 & 19353 \\
19314 & 65.67 & 87.45 & 19350 \\
19828 & 641.02 & 90.12 & \\
19349 & 63.17 & 87.85 & 19349
\end{tabular}

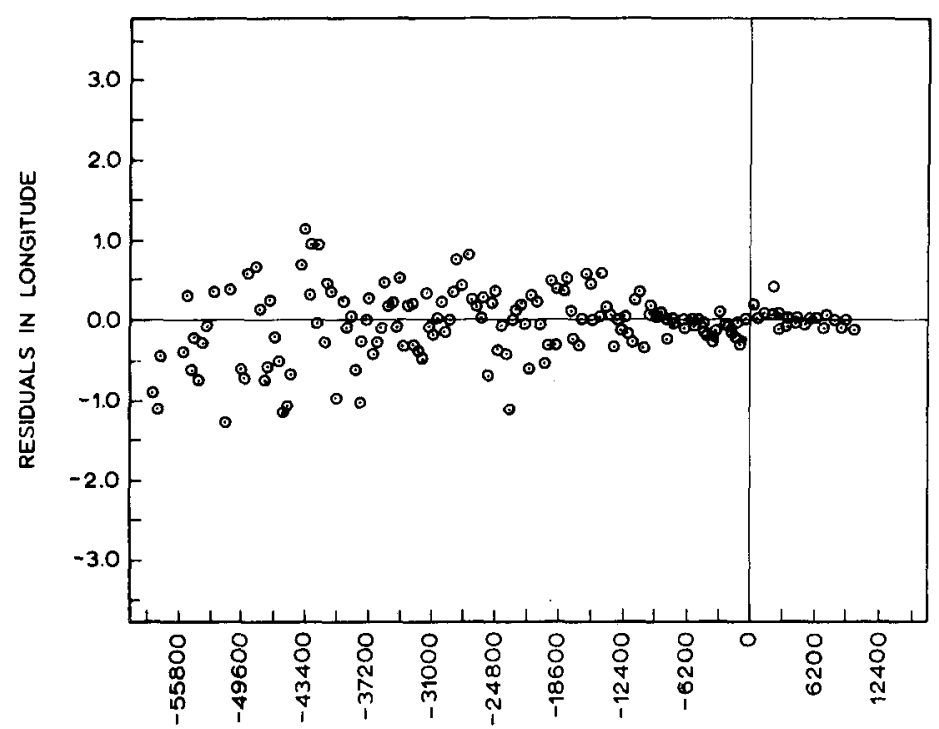

Fig. 4. Uranus 1781-1968 (JD-2430000.5).

elements of Neptune and a correction to the mass of Pluto. As previously mentioned the significant characteristic of past theories of the motion of Neptune has been their apparent failure to represent observations very far removed in time from the observations to which the constants of the theory were adjusted. That characteristic has been used as a test here in the attempt to determine the correct mass of Pluto.

Newcomb's masses were used initially for the Neptune orbit analysis and, for each 
TABLE V

Reciprocal mass of Pluto

\begin{tabular}{|c|c|c|c|c|}
\hline \multirow{2}{*}{$\begin{array}{l}\text { Reciprocal } \\
\text { mass test } \\
\text { values }\end{array}$} & \multicolumn{2}{|c|}{ Neptune $\Sigma v^{2}(\operatorname{arcsec})$} & \multicolumn{2}{|c|}{$1960-68$} \\
\hline & $\lambda$ & $\beta$ & $\lambda$ & $\beta$ \\
\hline 360000 & 32.01 & 21.87 & 64.47 & 0.33 \\
\hline 930000 & 30.72 & 24.00 & 3.71 & 0.06 \\
\hline 1500000 & 30.42 & 25.39 & 0.29 & 0.09 \\
\hline 2640000 & 30.22 & 26.51 & 0.51 & 0.14 \\
\hline 1812000 & 30.20 & 25.77 & 0.12 & 0.11 \\
\hline
\end{tabular}

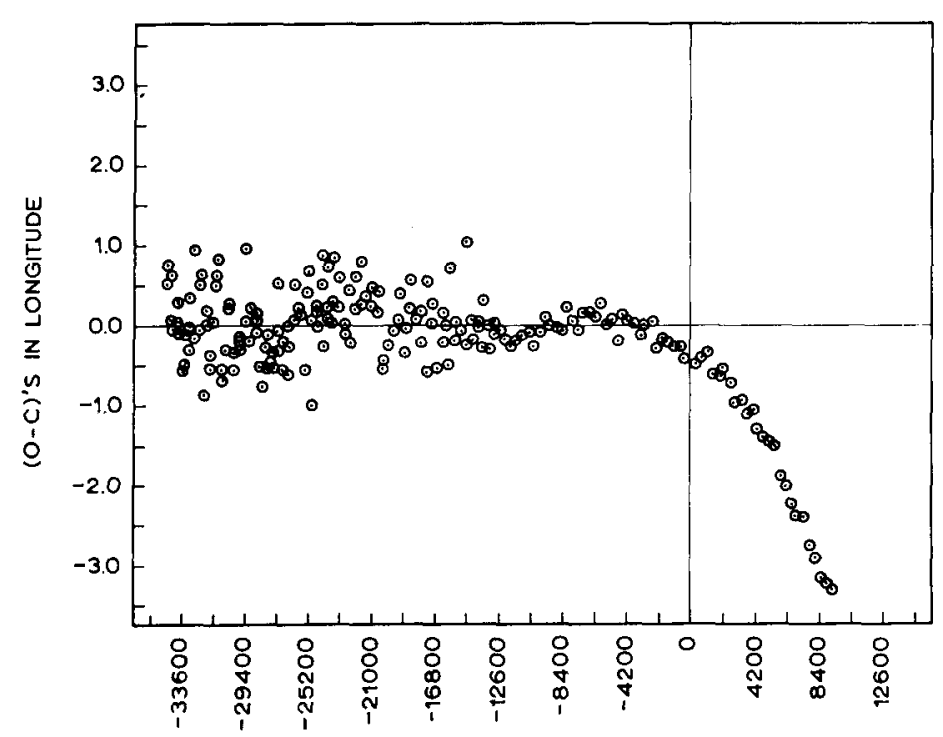

Fig. 5. Neptune, five outer planets (JD-2430000.5).

of the trial values of the mass of Pluto shown in Table V, the orbit of Neptune was fitted to observations from 1846 to 1938 . The observations from 1960-69 were then compared to the orbit and the sums of the squares of the residuals in longitude and latitude formed. Differentiating the equation of the parabola fitted through the first four longitude, $\Sigma v^{2}$ for the period $1960-68$ indicates a solution for the reciprocal mass of Pluto of 1812000 or 0.18 Earth masses. It should be recognized that this can be only a provisional result because it is sensitive to any systematic errors in the observations from 1960-68 and to a lesser degree in the observations prior to 1938. The solution has in fact been repeated incorporating all of the observations from 1938 up to 1968 , and the new system of masses derived here with a slight downward revision of the result to 0.17 Earth masses.

The improvement in the representation of the longitude observations achieved by 
TABLE VI

Reciprocal masses of the outer planets

$\begin{array}{lccl} & & \\ \text { Planet } & \text { Adopted value } & \text { New determination } \\ \text { Jupiter } & 1047.355 & 1047.366 \pm 0.006 \text { m.e. } \\ \text { Saturn } & 3501.6 & 3498.7 & \pm 0.2 \\ \text { Uranus } & 22869 & 22692 \quad \pm 33 \\ \text { Neptune } & 19314 & 19349 \quad \pm 28 \\ \text { Pluto } & 360000 & 1812000\end{array}$

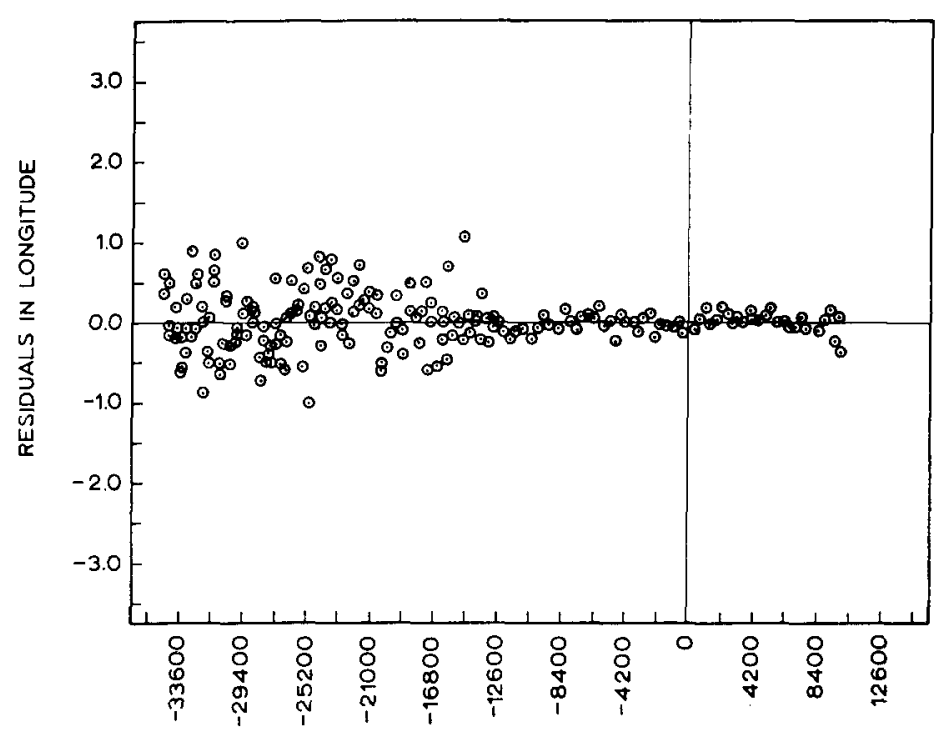

Fig. 6. Neptune 1846-1968 (JD-2430000.5).

the new orbit is shown in Figure 5 (the observations compared to the Eckert et al. theory) and Figure 6 (the observations compared to the present theory).

The new mass determinations compared to the presently adopted values are summarized in Table VI. It must be mentioned that in spite of these new mass determinations, the anomalous trends in the latitude residuals of Uranus, first reported by Newcomb and later investigated by Brouwer, still persist. Dr Jackson of the U.S. Naval Observatory is rediscussing the older observations of Neptune in hopes of removing some of the errors which are believed to be present. When the observations of Neptune have been rediscussed, a solution for a more definitive value of the mass of Pluto will be made.

\section{References}

Cohen, C. J., Hubbard, E. C., and Oesterwinter, C.: 1967, Astron. J. 72, 973.

Duncombe, R. L., Klepczynski, W. J., and Seidelmann, P. K.: 1968, Astron. J. 73, 830. 
Duncombe, R. L., Klepczynski, W. J., and Seidelmann, P. K.: 1971, Publ. Astron. Soc. Pacific 82, 916.

Eckert, W. J., Brouwer, D., and Clemence, G. M.: 1951, Astron. Pap. Am. Eph. 12.

Fiala, A. D.: 1968, Dissertation, Yale University.

Klepczynski, W. J.: 1969, Dissertation, Yale University.

Klepczynski, W. J., Seidelmann, P. K., and Duncombe, R. L.: 1970, Astron. J. 75, 739.

Newcomb, S.: 1898, Astron. Pap. Am. Eph. 6.

Newcomb, S.: 1898, Astron. Pap. Am. Eph. 7, part II.

O'Handley, D. A.: 1967, Dissertation, Yale University.

Seidelmann, P. K., Duncombe, R. L., and Klepczynski, W. J.: 1969, Astron. J. 74, 776.

Wylie, L. R.: 1942, Publ. U.S. Nav. Obs., Second Series, 15, pt. 1.

Zielenbach, J. W.: 1968, Dissertation, Georgetown University. 